\title{
Exercise and BP in obese children
}

Nathalie Farpour-Lambert and colleagues from the University Hospitals of Geneva, Switzerland have demonstrated that regular physical activity in obese children decreases systemic blood pressure (BP) and reduces arterial intima-media thickness (IMT) and arterial stiffness, which are markers of atherosclerosis. "The first signs of atherosclerosis appear early in obese children ... and the effects of physical activity on BP and early markers of atherosclerosis in obese children before puberty were unknown" explains Farpour-Lambert.

The increase in childhood obesity is a major public-health crisis and effective early interventions are needed to prevent cardiovascular disease (CVD) in this at-risk group. Previous research has shown that hypertension is strongly related to BMI in children and adolescents, with around half of obese pediatric patients developing hypertension. Physical activity can prevent and treat hypertension and CVD in adults, but its effects on obese children and adolescents have not been thoroughly investigated.

Farpour-Lambert et al. recruited 44 prepubertal obese children with a BMI over the 97th age-specific and sex-specific percentile with an average age of $8.9( \pm 1.5)$ years and 22 children within the same age group (mean age $8.5 \pm 1.5$ years) with a BMI within the normal range as controls. The investigators randomly assigned obese participants to either an exercise or a control group, with an equal distribution of girls and boys in each group. For the first 3 months, the obese children in the exercise group had three 60-min training sessions per week whereas children in the control group remained relatively inactive. In the second 3-month period, children in both groups exercised twice per week. "We chose a modified cross-over design as we felt that it was unethical to leave obese children without treatment for more than 3 months," explains Farpour-Lambert. The researchers assessed changes at 3 and 6 months in BP, arterial IMT and stiffness, BMI, cardiorespiratory fitness ( $\mathrm{VO}_{2}$ max), and levels of various biological markers including plasma insulin and $\mathrm{HDL}$ cholesterol.

Children assigned to the exercise intervention had good compliance (83\%) and adherence (86\%) to the program. Farpour-Lambert et al. observed that, after 3 months, the exercise group showed significant decreases in systemic $\mathrm{BP}$, ranging from -7 to $-12 \mathrm{mmHg}$ for systolic $\mathrm{BP}$ and from -2 to $-7 \mathrm{mmHg}$ for diastolic BP. Additionally, exercise was associated with reductions in abdominal fat $(-4.2 \%)$ and whole body fat $(-3.6 \%)$, and significant increases in fat-free mass $(+4.6 \%)$ and $\mathrm{VO}_{2}$ $\max (+6 \%)$. The researchers observed further changes in the obese children after 6 months, with a significant decrease in arterial stiffness as well as stabilization of the arterial IMT. Furthermore, in the exercise group, the proportion of obese children with hypertension decreased from $50 \%$ at baseline to $37 \%$ at 3 months, and to $29 \%$ at 6 months.

"Schools should provide physical education lessons at least 3 times 60 min per week," says Farpour-Lambert who suggests that the sessions should be adapted for obese children to encourage their participation. "CVD risk factors track from childhood to adulthood," adds Farpour-Lambert and she explains that promotion of physical activity programs would help prevent premature development of atherosclerosis and reduce CVD risks in young obese children.

Katrina Ray

Original article Farpour-Lambert, N. J. et al. Physical activity reduces systemic blood pressure and improves early markers of atherosclerosis in pre-pubertal obese children. J. Am. Coll. Cardiol. 54, 2396-2406 (2009) 